\section{Retroperitoneal, Mediastinal, and Subcutane- ous Emphysema as a Complication of Routine Upper Gastrointestinal Endoscopy}

We report here the case of a 62 -year-old woman presenting with a massive retroperitoneal, mediastinal, and subcutaneous emphysema (RMSCE) following a routine upper gastrointestinal fiberoptic endoscopy, with no obvious evidence of a site of perforation. Endoscopy (Olympus XQ) was performed for work-up of dyspepsia in blind swallow fashion; the esophagus, stomach and duodenum were normal. The antral mucosa was hyperemic, a biopsy was taken. Shortly after endoscopy, the patient became restless, and complained of neck pain and abdominal discomfort. Physical examination revealed subcutaneous emphysema, and a radiograph of the chest and abdomen (Figures 1, 2) showed air in the subcutaneous tissue and a massive pneumomediastinum and pneumo-retroperitoneum. An upper gastrointestinal tract study and CT of the chest and abdomen with gastrographin were performed, but did not reveal any evidence of perforation or contrast leakage; the CT confirmed RMSCE. The patient was treated conservatively with

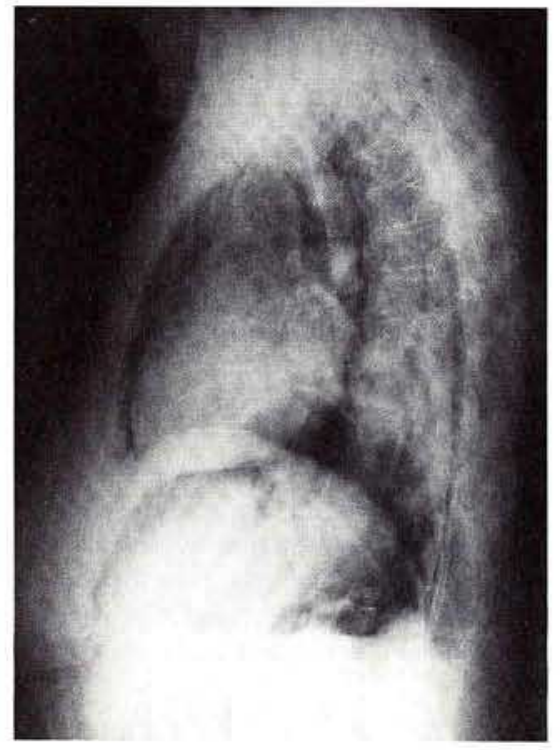

Figure 1:

Lateral chest radiograph. A large amount of subdiaphragmatic pneumoretroperitoneal air, and pneumomediastinum, are seen. 
intravenous fluids and antibiotics, and he improved gradually and was discharged 20 days after the upper gastrointestinal endoscopy.

RMSCE without obvious evidence of a perforation site following routine upper gastrointestinal endoscopy is a rare complication that has been described only once in the literature (1). However, several reports have described pneumomediastinum alone (2), or retroperitoneal emphysema alone (3), with no demonstrable perforation. The unusual distribution of the air in our patient might have been caused by air disseminating through the antral wall at a point of mucosal weakness (due to the biopsy taken from the antrum), and then spreading along the vascular sheaths to the serosal surface, rupturing into the peritoneal cavity. Another possibility, proposed by Macklin (4), is that air leaks through numerous minute openings in the base of the alveolar spaces, extending into perivascular sheaths (5) after an artificial rise in the airway pressure due to excessive air insufflation into the stomach. The air could then penetrate along these sheaths to the hilum of the lung, creating mediastinal and eventually subcutaneous emphysema, on the one hand, and spread through the esophageal hiatus to the retroperitoneum, on the other hand $(4,5)$. As far as treatment is concerned, our view is that, once perforation has been excluded, a more conservative approach might be indicated.

\section{Z. Fireman I, 3 , J. Bar-Ziv², B. Lurie I}

${ }^{1}$ Gastroenterology Unit and ${ }^{2}$ Radiology Department

Golda Medical Center, Hasharon Hospital

Petah-Tiqva and the Sackler School of Medicine

Tel-Aviv University, Israel;

${ }^{3}$ Gastrointestinal Department, Hillel Yaffe Medical Center

P. O. Box 169, Hadera 31800 , Israel

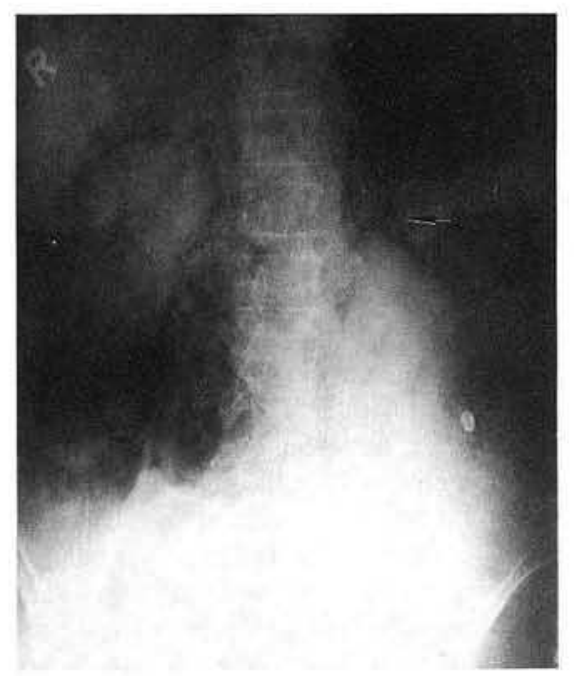

Figure 2: Anteroposterior view of the abdomen Pneumoretroperitoneum, with air surrounding the kidney and the left adrenal (arrow), is seen.

\section{References}

1. Girardi A, Piazza I, Giunta G, Pappagallo G: Retroperitoneal, mediastinal and subcutaneous emphysema as a complication of routine upper gastro-intestinal endoscopy. Endoscopy 1990; 22: 83-84.

2. Katz D, Cano $R$, Antonello M: Benign air dissection of the esophagus and stomach at fiberoptic endoscopy. Gastrointest. Endosc. 1972; 19: 72 .

3. Martin WC, Nagai N, Diuer W, Texter EC, Jr.: Benign retroperitoneal emphysema associated with upper gastrointestinal endoscopy. Arch. Int. Med. 1978; 138: 759-768.

4. Macklin CC: Transport of air along sheaths of pulmonic blood vessels from alveoli to mediastinum. Arch. Intern. Med. 1939; 64: 913-919.

5. Schulman A, Fattar S, Van der Spuy SW, et al.: Air in unusual places: some causes and ramifications of pneumomediastinum. Clin. Radiol. 1982; 33: 301-306. 